\title{
How quantum bound states bounce and the structure it reveals
}

\author{
D. Lee ${ }^{\mathrm{a}}$ and M. Pine \\ Department of Physics, North Carolina State University, Raleigh, NC 27695, USA
}

Received: 26 October 2010 / Revised: 4 February 2011

Published online: 21 March 2011

(c) The Author(s) 2011. This article is published with open access at Springerlink.com

Communicated by H. Wittig

\begin{abstract}
We investigate how quantum bound states bounce from a hard surface. Our analysis has applications to $a b$ initio calculations of nuclear structure and elastic deformation, energy levels of excitons in semiconductor quantum dots and wells, and cold atomic few-body systems on optical lattices with sharp boundaries. We develop the general theory of elastic reflection for a composite body from a hard wall. On the numerical side we present $a b$ initio calculations for the compression of alpha particles and universal results for two-body states. On the analytical side we derive a universal effective potential that gives the reflection scattering length for shallow two-body states.
\end{abstract}

In this letter we consider the elastic scattering of quantum bound states from a hard surface. Elastic scattering from a hard surface provides direct information about the structure of the bound state and how it behaves under spatial confinement and compression. Spatially confined bound states are interesting quantum systems with important practical applications. For example in quantum dots and wells, electron-hole pairs are confined to the interior of nanoscale semiconductor structures. The size and shape of the structure strongly influences the density of exciton states, thereby allowing the control of current tunneling as well as photon absorption and emission. See for example refs. [1-4]. In our analysis we present universal results for direct band-gap semiconductors, relating exciton energies to the binding energy at infinite volume, the effective masses of the electrons and holes, and the geometry of the nanostructure.

Confined systems can also be produced in cold atomic experiments. A quantum well can be constructed using an optical lattice with additional lasers focused to produce sharp repulsive boundaries. Similar ideas have been proposed for quantum billiards systems [5]. Since a flat hardwall boundary has no dimensionful scale, reflection from the wall probes the universal physics of few-body systems at large scattering length. For example the dimer-wall reflection scattering length for a shallow dimer is directly proportional to the two-particle scattering length. In the following we will calculate the universal constant of proportionality between these two scattering lengths.

We consider a non-relativistic bound state in $d$ spatial dimensions with mass $M$. The number of constituent particles comprising the bound state is arbitrary. The bound

\footnotetext{
a e-mail: dean_lee@ncsu.edu
}

state scatters elastically against a flat hard-wall boundary. Let $X$ be the distance from the wall to the center of mass of the bound state. We choose the inertial frame where all components of momentum parallel to the wall surface are zero and construct a standing-wave solution with momenta $\pm p$ perpendicular to the wall. We denote the reflection phase shift as $\delta(p)$, and define the reflection radius as $R(p)=-\delta(p) / p$. This corresponds with the distance between the wall and the closest node of the asymptotic standing wave, $\Psi_{p}(X) \propto \sin [p X+\delta(p)]$.

The reflection scattering process is analogous to $S$-wave scattering in three dimensions. We can therefore use the effective range expansion

$$
p \cot \delta(p)=-\frac{1}{a_{R}}+\frac{1}{2} r_{R} p^{2}-\mathcal{P}_{R} p^{4}+\cdots,
$$

where $a_{R}$ is the scattering length, $r_{R}$ is the effective range, and $\mathcal{P}_{R}$ is the shape parameter. We note that $a_{R}$ equals the reflection radius at threshold, $a_{R}=\lim _{p \rightarrow 0} R(p)$. If the bound-state structure is completely rigid then $R(p)=a_{R}$ for all $p$. For realistic systems, however, compression of the bound state increases with collision energy. Hence $R(p)$ decreases monotonically with $p$, and the rate of decrease indicates the compressibility of the bound state under unilateral stress. The reflection radius is directly related to the energy of the bound state under spatial confinement. For a $d$-dimensional cube with length $L$ and vanishing Dirichlet boundaries, the energy of the lowest confined mode is

$$
E(L)=\frac{p^{2}(L) \cdot d}{2 M}+O\left(L^{-4}\right), \quad p(L)=\frac{\pi}{L-2 R[p(L)]} .
$$

This formula arises from the sine function profile with halfwavelength $L-2 R[p(L)]$ in each dimension. It can be used 
Table 1. Momenta and reflection radii for an alpha particle confined to a cube of length $L$.

\begin{tabular}{|c|c|c|}
\hline$L$ & $p(L)$ & $R[p(L)]$ \\
\hline \hline $11.8 \mathrm{fm}$ & $81(9) \mathrm{MeV}$ & $2.1(4) \mathrm{fm}$ \\
$9.9 \mathrm{fm}$ & $97(10) \mathrm{MeV}$ & $1.6(3) \mathrm{fm}$ \\
$7.9 \mathrm{fm}$ & $118(10) \mathrm{MeV}$ & $1.3(2) \mathrm{fm}$ \\
\hline
\end{tabular}

to determine the reflection radius as a function of momentum. The $O\left(L^{-4}\right)$ error estimate contains corrections due to double-wall collision effects near the wall intersections.

It is not possible to construct a hard surface for protons and neutrons in nuclear-physics experiments. Therefore it may seem that the current discussion has no direct connection with nuclear physics. A similar critique could be made of Lüscher's analysis of periodic boundaries in finite cubic volumes $[6,7]$. However Lüscher's analysis now provides the theoretical framework for numerous calculations in lattice quantum chromodynamics [8-10]. The point is that $a b$ initio numerical calculations play an increasingly important role in nuclear theory. In some cases the numerical calculations investigate phenomena directly observed in experiments. In other cases the calculations probe new physics not accessible in experiments. Similar to temperature and chemical potential, different spatial boundaries provide tunable control parameters for the numerical calculations.

We now briefly discuss one example from nuclear physics. There has been much interest in alpha-particle clusters inside nuclei such as carbon-12 [11-13]. Very recently there have been $a b$ initio lattice calculations of the low-lying states of carbon-12 using effective field theory [14]. In addition to the ground state and excited spin-2 state, these calculations find for the first time the Hoyle resonance responsible for carbon formation at stellar temperatures. The lattice data suggests the presence of correlated alpha clusters. Furthermore it appears that the alpha clusters themselves are compressed in size when bound in nuclei. This would be interesting since there are no lowenergy resonances of the alpha particle and little experimental data on the compression of alpha particles.

We have calculated the energy of an alpha particle at leading order in chiral effective field theory when confined to a cubic box with vanishing Dirichlet boundaries. We use the same lattice action, algorithms, and codes as in ref. [14]. The boundaries are imposed by adding an essentially infinite potential energy at the wall boundaries. We note that the ultraviolet divergences are independent of long-distance boundary conditions, and so no new renormalization counterterms are needed. We only summarize the results here, and the numerical details of this calculation will be discussed in a forthcoming publication [15].

At leading order we have calculated the energy for cubic boxes with lengths $L=11.8 \mathrm{fm}, 9.9 \mathrm{fm}$, and $7.9 \mathrm{fm}$. The results are shown in table 1 . The error bars in table 1 are one standard deviation estimates which include both Monte Carlo statistical errors and uncertainties due to extrapolation at large Euclidean time. The reflection radii in table 1 can be compared with the root-mean-square
(RMS) matter radius of the alpha particle. At leading order we find the RMS matter radius for pointlike nucleons to be $1.53(4) \mathrm{fm}$.

At low momenta the reflection radius of the alpha particle is somewhat larger than the RMS matter radius. However, we see a substantial decrease of the alpha particle reflection radius with increasing momentum. This data suggests that the alpha particles do compress quite readily with pressure. This appears consistent with the observation that alpha clusters are compressed in size when bound in nuclei. Much more numerical work is planned to study alpha particles and other nuclei under confinement pressure.

We now focus on the case of two-body bound states. The masses of the two constituent particles will be denoted $m_{1}$ and $m_{2}$, and $\mu$ is the reduced mass, $\mu=\left(m_{1}^{-1}+\right.$ $\left.m_{2}^{-1}\right)^{-1}$. We assume the two constituent particles are distinguishable and so the quantum statistics and spin are irrelevant. For the case of electron-hole pairs, $m_{1}$ and $m_{2}$ correspond with the effective masses of the electrons and holes around zero-momentum minima. Our analysis applies to the case of Wannier excitons where the exciton size is significantly larger than the Coulomb screening length.

Let $E_{B}$ be the energy of the dimer at infinite volume, and $\kappa_{B}$ be the binding momentum defined by the relation $E_{B}=-\kappa_{B}^{2} /(2 \mu)$. We assume that the bound dimer has zero orbital angular momentum and let $a_{B}$ be the $S$-wave scattering length for the two constituent particles. The total spin of the bound state is irrelevant to our analysis so long as there is negligible partial-wave mixing. We consider the shallow-binding limit where $a_{B}$ is much larger than the range of the interaction. In this limit $\kappa_{B}=a_{B}^{-1}$, and the low-energy physics of shallow dimers is independent of the short-distance details of the interaction. It follows that the dimer-wall reflection phase shift is a universal function of the dimensionless ratio $p / \kappa_{B}$. In the following discussion we present the form of this universal function.

We have calculated the dimer-wall reflection phase shift for the one- and three-dimensional systems numerically using a Hamiltonian lattice formalism. More details on this lattice formalism can be found in ref. [16]. The two-particle interaction is implemented as an attractive single-site interaction. Two parallel hard-wall boundaries are placed a distance $L$ apart, and we set the momentum of the dimer parallel to the wall equal to zero. The confinement energy is measured as a function of $L$ using sparsematrix eigenvector methods to determine the reflection phase shift. Calculations are repeated several times using successively smaller lattice spacings to extrapolate to the continuum limit and determine universal results in the shallow-binding limit. For the three-dimensional case we also perform an infinite-size extrapolation in the dimensions perpendicular to the wall. A large number of separate calculations are required to perform these extrapolations, and the details of each calculation are described in a forthcoming publication [15].

Figure 1 shows the one-dimensional results for the reflection radius versus the dimer kinetic energy, $E_{K}$, in 


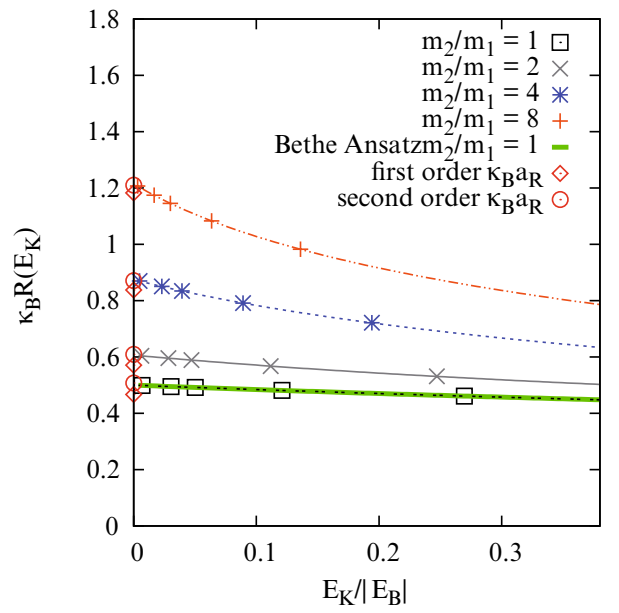

Fig. 1. One-dimensional lattice results for the reflection radius versus the dimer kinetic energy in the shallow-binding limit. Also shown are Bethe Ansatz results for $m_{2} / m_{1}=1$ and firstand second-order results for the expansion of $\kappa_{B} a_{R}$ described later in the text.

Table 2. Coefficients of the effective range expansion for onedimensional dimer-wall scattering.

\begin{tabular}{|c|c|c|c|}
\hline$m_{2} / m_{1}$ & $\kappa_{B} a_{R}$ & $\kappa_{B} r_{R}$ & $\kappa_{B}^{3} P_{R}$ \\
\hline \hline 1 & $0.4999(2)$ & $0.005(7)$ & $0.002(3)$ \\
2 & $0.6065(2)$ & $-0.074(2)$ & $-0.006(2)$ \\
4 & $0.8747(2)$ & $0.115(2)$ & $0.006(2)$ \\
8 & $1.2149(2)$ & $0.460(2)$ & $0.008(2)$ \\
\hline
\end{tabular}

the shallow-binding limit. The results are presented in dimensionless combinations, $\kappa_{B} R\left(E_{K}\right)$ versus $E_{K} /\left|E_{B}\right|$. We show results for several different mass ratios, $m_{2} / m_{1}$. As expected the reflection radius decreases monotonically with energy. One interesting feature is the dependence on the mass ratio. For large $m_{2} / m_{1}$ the reflection radius is rather large at small energies while significantly decreasing with energy. We explain this mass ratio dependence later in our discussion.

For the case $m_{2} / m_{1}=1$, the one-dimensional problem is integrable and can be solved exactly using the Bethe Ansatz. From the Bethe Ansatz we get

$$
\begin{aligned}
p \cot \delta(p) & =-2 \kappa_{B}, \\
\kappa_{B} R\left(E_{K}\right) & =\frac{1}{2} \sqrt{\frac{\left|E_{B}\right|}{E_{K}}} \tan ^{-1} \sqrt{\frac{E_{K}}{\left|E_{B}\right|}} .
\end{aligned}
$$

As shown in fig. 1, the agreement between the lattice calculations and Bethe Ansatz solution is very good. Figure 1 also shows the first- and second-order analytic results for the expansion of $\kappa_{B} a_{R}$ described later in our discussion.

The coefficients of the effective range expansion for the one-dimensional dimer-wall scattering are given in table 2. The error estimates are from least-squares fitting used in the lattice extrapolation and the effective range expansion. As one can see from eq. (3), the Bethe Ansatz

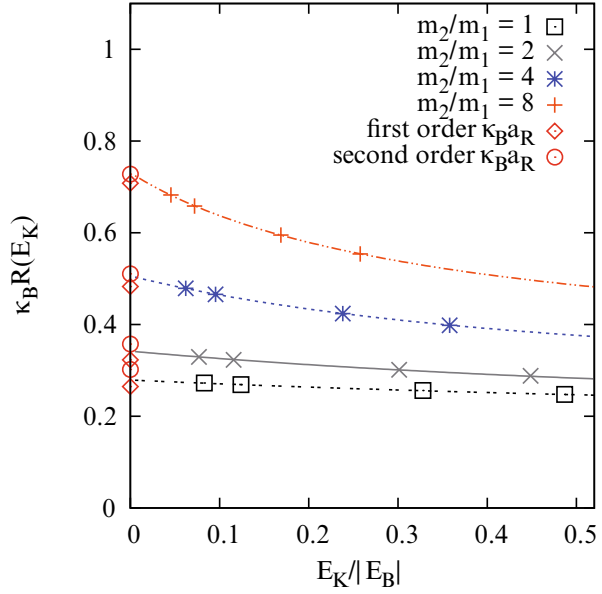

Fig. 2. Three-dimensional lattice results for the reflection radius versus the dimer kinetic energy in the shallow-binding limit. Also shown are first- and second-order results for $\kappa_{B} a_{R}$ described later in the text.

Table 3. Coefficients of the effective range expansion for threedimensional dimer-wall scattering.

\begin{tabular}{|c|c|c|c|}
\hline$m_{2} / m_{1}$ & $\kappa_{B} a_{R}$ & $\kappa_{B} r_{R}$ & $\kappa_{B}^{3} P_{R}$ \\
\hline \hline 1 & $0.279(8)$ & $-0.38(9)$ & $-0.02(2)$ \\
2 & $0.342(8)$ & $-0.44(6)$ & $-0.02(2)$ \\
4 & $0.506(8)$ & $-0.27(4)$ & $-0.02(1)$ \\
8 & $0.731(8)$ & $0.03(2)$ & $-0.005(5)$ \\
\hline
\end{tabular}

gives $\kappa_{B} a_{R}=1 / 2$ for $m_{2} / m_{1}=1$, with all other higher coefficients equal to zero. This is in full agreement with the lattice results. The results are in principle universal and can be checked using other theoretical methods or perhaps experiments such as cold atomic dimers on a onedimensional optical lattice with sharp boundaries.

Figure 2 shows the reflection radius versus the dimer kinetic energy for the three-dimensional system in the shallow-binding limit. Again the results are presented in dimensionless combinations, $\kappa_{B} R\left(E_{K}\right)$ versus $E_{K} /\left|E_{B}\right|$. The reflection radius is nearly a factor of two smaller than in the one-dimensional case. This is reasonable considering the difference between compression of a one-dimensional object versus compression of a three-dimensional sphere along one direction. As in the one-dimensional case, we see the same dependence on the mass ratio. At large $m_{2} / m_{1}$ the reflection radius is large at small energies while becoming substantially smaller with increasing energy. Figure 2 also shows the first- and second-order results for the asymptotic expansion of $\kappa_{B} a_{R}$ described later in our discussion.

The coefficients of the effective range expansion for the three-dimensional system are given in table 3 . One immediate application of our three-dimensional results would be to calculate the reflection phase shift of the deuteron from $a b$ initio lattice calculations using chiral effective field theory. In addition to verifying the universal coefficients in table 3 , it should be possible to measure the spin 
dependence of the reflection phase shift resulting from the $D$-wave component of the deuteron wave function.

We now discuss the analytic calculation of the reflection scattering length for a shallow dimer. The calculation we present uses the principles of effective field theory but covers somewhat unfamiliar territory. Namely, our system is inhomogeneous and there is no obvious small expansion parameter.

The method we use is to apply an adiabatic approximation for the center-of-mass motion and generate an asymptotic expansion for the long-distance part of the effective potential. We find that this process generates an expansion for $\kappa_{B} a_{R}$ with convergence controlled by an expansion parameter of size $e^{-2 \kappa_{B} a_{R}}$. The derivation and full details of this expansion up to second order will be given in a forthcoming paper [15]. We summarize the main method and results here.

Let the coordinates of the two constituent particles be $\vec{r}_{1}$ and $\vec{r}_{2}$. The particles have a short-range attractive interaction, and we take the Hamiltonian to be

$$
H=-\frac{1}{2 m_{1}} \vec{\nabla}_{r_{1}}^{2}-\frac{1}{2 m_{2}} \vec{\nabla}_{r_{2}}^{2}+C_{B} \bar{\delta}^{(d)}\left(\vec{r}_{1}-\vec{r}_{2}\right)
$$

where $\bar{\delta}^{(d)}$ is a regulated $d$-dimensional delta function. The coefficient $C_{B}$ is tuned to produce a bound state with energy $E_{B}$ at infinite volume. Let $\vec{r}=\vec{r}_{1}-\vec{r}_{2}$ be the relative separation between the particles. For any fixed center-of-mass coordinate the dependence on the relative coordinate is given by

$$
H_{\mathrm{rel}}=-\frac{1}{2 \mu} \vec{\nabla}_{r}^{2}+C_{B} \bar{\delta}^{(d)}(\vec{r}) .
$$

To calculate the reflection scattering length it suffices to compute the dimer-wall scattering in the limit $E_{K} \ll\left|E_{B}\right|$. For this low-energy limit we use an adiabatic expansion for the center-of-mass motion. This technique is conceptually similar to the adiabatic hyperspherical approximation [17-19]. For each $X$ we keep only the ground state for $H_{\text {rel }}$ satisfying the hard-wall boundary condition. Contributions from higher excitations in $H_{\text {rel }}$ are suppressed by powers of $E_{K} /\left|E_{B}\right|$. Therefore these higher excitations contribute to higher-order coefficients in the effective range expansion but make no contribution to the reflection scattering length.

For each $X$ let $\psi_{X}(\vec{r})$ be the normalized ground-state wave function of $H_{\text {rel }}$ satisfying the hard-wall boundary constraint. The low-energy effective Hamiltonian is

$$
H_{\mathrm{eff}}=-\frac{1}{2 M} \frac{\partial^{2}}{\partial X^{2}}+V(X)+T(X),
$$

where $V(X)$ is the adiabatic potential,

$$
V(X)=\left\langle\psi_{X}\left|H_{\text {rel }}\right| \psi_{X}\right\rangle
$$

and $T(X)$ is the diagonal adiabatic correction,

$$
T(X)=-\frac{1}{2 M}\left\langle\psi_{X}\left|\frac{\partial^{2}}{\partial X^{2}}\right| \psi_{X}\right\rangle .
$$

We note that the cross-term involving the expectation value of $\psi_{X}$ for one derivative with respect to $X$ vanishes due to the fixed normalization of $\psi_{X}$.

Let us now define the length scales $r_{+}(X)=2 M X / m_{1}$ and $r_{-}(X)=2 M X / m_{2} \cdot r_{+(-)}(X)$ is twice the distance from the wall to $\vec{r}_{1(2)}$ when the other particle touches the wall. In other words $\psi_{X}(\vec{r})$ must vanish when the component of $\vec{r}$ perpendicular to the wall equals $r_{+}(X) / 2$ or $-r_{-}(X) / 2$. For large $X$ we can generate an asymptotic expansion for $V(X)$ and $T(X)$ in powers of $e^{-\kappa_{B} r_{+}(X)}$ and $e^{-\kappa_{B} r_{-}(X)}$

At zeroth order we recover the infinite-volume result, $V^{(0)}(X)+T^{(0)}(X)=E_{B}$. At first order the effective potential for the one-dimensional case is

$$
\begin{aligned}
& T^{(1)}(X)+V^{(1)}(X)= \\
& \quad \frac{\kappa_{B}^{2} M^{2}}{m_{1} m_{2}}\left[\frac{e^{-\kappa_{B} r_{+}(X)}}{m_{1}}+\frac{e^{-\kappa_{B} r_{-}(X)}}{m_{2}}\right] .
\end{aligned}
$$

In three dimensions the result is

$$
\begin{aligned}
& T^{(1)}(X)+V^{(1)}(X)= \\
& \quad \frac{\kappa_{B} M}{2 m_{1} m_{2} X}\left[e^{-\kappa_{B} r_{+}(X)}+e^{-\kappa_{B} r_{-}(X)}\right] .
\end{aligned}
$$

From these effective potentials it is straightforward to compute the first-order result for the reflection scattering length. This process can be carried forward to any order. The net result is an expansion with an expansion parameter of size $e^{-\kappa_{B} r_{ \pm}\left(a_{R}\right)} \leq e^{-2 \kappa_{B} a_{R}}$. The larger $\kappa_{B} a_{R}$, the faster the convergence of the expansion. First- and secondorder results for the one-dimensional system are shown in fig. 1, and results for the three-dimensional system are shown in fig. 2. In each case the agreement with lattice results is consistent with third-order corrections of size $\leq e^{-6 \kappa_{B} a_{R}}$.

We now finally address what happens in the limit $m_{2} / m_{1} \rightarrow \infty$. Consider the limit $m_{2} \rightarrow \infty$ with $m_{1}$ held fixed. In this limit the effective potential converges to a non-vanishing finite-valued function, while the mass of the dimer grows with $m_{2}$. One can check this explicitly for the expressions in eq. (10) and eq. (11). Given the exponential tail of the effective potential, the reflection radius near threshold has a logarithmic dependence on $m_{2} / m_{1}$. An immediate application of these results would be to verify the logarithmic $m_{2} / m_{1}$ threshold dependence in various physical systems. This effect should be prominent for halo nuclei with a heavy core and one satellite nucleon. It should also be seen in quantum dots and wells for semiconductors with a large ratio between hole and electron effective masses. It could also be reproduced with heterogeneous cold atomic dimers consisting of one heavy and one light alkali atom.

In this letter we have discussed a number of phenomena relating to the elastic scattering of quantum bound states from a hard surface. We have emphasized processes of universal character which can be seen in several different systems. The applications appear numerous, ranging from experimental predictions for quantum dots and wells 
to numerical calculations of nuclear structure. In our analysis we have introduced some theoretical techniques which may be useful for describing the effective field theory of other inhomogeneous systems.

The authors are grateful to Chris Greene, Hans-Werner Hammer, Ed Marti, and Andreas Wieck for useful discussions. Financial support from the U.S. Department of Energy and NC State GAANN Fellowship is acknowledged. Computational resources are provided by the Jülich Supercomputing Centre at Forschungszentrum Jülich.

Open Access This article is distributed under the terms of the Creative Commons Attribution Noncommercial License which permits any noncommercial use, distribution, and reproduction in any medium, provided the original author(s) and source are credited.

\section{References}

1. T. Takagahara, K. Takeda, Phys. Rev. B 46, 15578 (1992).

2. J.Y. Marzin, J.M. Gérard, A. Izraël, D. Barrier, G. Bastard, Phys. Rev. Lett. 73, 716 (1994).

3. S. Pfalz, R. Winkler, T. Nowitzki, D. Reuter, A.D. Wieck, D. Hägele, M. Oestreich, Phys. Rev. B 71, 165305 (2005).
4. L. He, G. Bester, A. Zunger, Phys. Rev. Lett. 94, 016801 (2005).

5. S. Montangero, D. Frustaglia, T. Calarco, R. Razio, EpL 88, 30006 (2009).

6. M. Lüscher, Commun. Math. Phys. 104, 177 (1986).

7. M. Lüscher, Commun. Math. Phys. 105, 153 (1986).

8. NPLQCD Collaboration (S.R. Beane, P.F. Bedaque, K. Orginos, M.J. Savage), Phys. Rev. D 73, 054503 (2006) hep-lat/0506013.

9. S.R. Beane, P.F. Bedaque, K. Orginos, M.J. Savage, Phys. Rev. Lett. 97, 012001 (2006) hep-lat/0602010.

10. V. Bernard, M. Lage, U.-G. Meißner, A. Rusetsky, JHEP 08, 024 (2008) arXiv:0806.4495.

11. A. Tohsaki, H. Horiuchi, P. Schuck, G. Ropke, Phys. Rev. Lett. 87, 192501 (2001) nucl-th/0110014.

12. M. Chernykh, H. Feldmeier, T. Neff, P. von NeumannCosel, A. Richter, Phys. Rev. Lett. 98, 032501 (2007).

13. Y. Suzuki et al., Phys. Lett. B 659, 160 (2008) nucl-th/ 0703001.

14. E. Epelbaum, H. Krebs, D. Lee, U.-G. Meißner, arXiv: 1101.2547.

15. D. Lee, M. Pine, in preparation.

16. D. Lee, Prog. Part. Nucl. Phys. 63, 117 (2009) arXiv: 0804.3501 [nucl-th].

17. J. Macek, J. Phys. B 1, 831 (1968).

18. C.D. Lin, Phys. Rep. 257, 1 (1995).

19. B.D. Esry, C.D. Lin, C.H. Greene, Phys. Rev. A 54, 394 (1996). 